Table 1. Number of Press Articles Reporting Infections Caused by CRAB and CRE in Brazil, Distributed in Periods and Country's Macroregions

\begin{tabular}{lcccccc}
$\begin{array}{l}\text { Subject } \\
\text { and Date }\end{array}$ & North & Northeast & Middle-West & Southeast & South & Total \\
\hline CRAB & & & & & & \\
\hline $2006-2010$ & $\ldots$ & $\ldots$ & $\ldots$ & $\ldots$ & $\ldots$ & $\ldots$ \\
\hline $2011-2015$ & $\ldots$ & 1 & 4 & 4 & $\ldots$ & 9 \\
\hline $2016-2018$ & $\ldots$ & $\ldots$ & $\ldots$ & $\ldots$ & $\ldots$ & $\ldots$ \\
\hline CRE & & & & & & \\
\hline $2006-2010$ & $\ldots$ & 2 & 2 & 2 & 4 & 10 \\
\hline $2011-2015$ & $\ldots$ & 13 & 8 & 19 & 13 & 53 \\
\hline $2016-2018$ & $\ldots$ & 4 & 4 & 5 & $\ldots$ & 13 \\
\hline
\end{tabular}

Note. CRAB, carbapenem-resistant Acinetobacte baumanii complex. CRE, carbapenemresistant enterobacteriaceae.

resolution that banned over-the-counter sales of antimicrobials in drugstores. That measure, focusing on outpatients, was not likely to succeed in controlling CRE spread in hospitals. Nevertheless, there was a substantial decrease in antimicrobial sales in private drugstores in response to public opinion. ${ }^{10}$ The extent to which this decrease has influenced or will influence antimicrobial resistance is a matter for further research.

We did not perform quantitative or qualitative analysis of press articles content. Those approaches were beyond our scope, but they open interesting venues for investigation. The Brazilian case shed lights on the press media and public response to epidemiologically complex issues, such as HAIs and AMR. It also reinforces the importance of public communication for the practice of healthcare epidemiology. Messages to the general public must be delivered in clear, objective language and with evidence-grounded information. If we avoid negligence and panic, public awareness can support effective interventions for infection prevention and control.
Acknowledgments. Partial results of this study were presented (among other studies) in P.Z.A.C. doctoral thesis in the Postgraduate Program in Tropical Infections, Botucatu School of Medicine, São Paulo State University (UNESP), City of Botucatu, São Paulo State, Brazil.

Financial support. F.F.C. received a student grant from the Brazilian Council for Scientific Development and Technology (CNPq), with CMCBF as advisor.

Conflicts of interest. All authors state that they have no conflict of interest regarding this study.

\section{References}

1. Shallcross LJ, Berin M, Roe J, Noursadeghi M. Are the public getting the message about antimicrobial resistance? Arch Public Health 2015; 73:55.

2. Marcel JP, Alfa M, Baquero F, et al. Healthcare-associated infections: think globally, act locally. Clin Microbiol Infect 2008;14:895-907.

3. Frieden TR. Six components necessary for effective public health program implementation. Am J Public Health 2014;104:17-22.

4. Allegranzi B, Bagheri Nejad S, Combescure C, et al. Burden of endemic healthcare-associated infection in developing countries: systematic review and meta-analysis. Lancet 2011;377:228-241.

5. Wakefield MA, Loken B, Hornik RC. Use of mass media campaigns to change health behaviour. Lancet 2010;376:1261-1271.

6. Bou-Karroum L, El-Jardali F, Hemadi N, et al. Using media to impact health policy making: an integrative systematic review. Implement Sci 2017;12:52.

7. Rossi F. The challenges of antimicrobial resistance in Brazil. Clin Infect Dis 2011;52:1138-1143.

8. Padoveze MC, Melo S, Bishop S, Poveda VB, Fortaleza CMCB. Public policies on healthcare-associated infections: a Brazil and UK case study. Rev Saude Publica 2017;51:119.

9. Padoveze MC, Fortaleza CM. Healthcare-associated infections: challenges to public health in Brazil. Rev Saude Publica 2014;48:995-1001.

10. Moura ML, Boszczowski I, Mortari N, et al. The impact of restricting overthe-counter sales of antimicrobial drugs: preliminary analysis of national data. Medicine (Baltimore) 2015;94(38):e1605.

\title{
Monitoring healthcare professionals after monkeypox exposure: Experience from the first case imported to Asia
}

\author{
Win Mar Kyaw MPH${ }^{1}$, Shawn Vasoo MRCP ${ }^{2,3}$, Hanley Jian An Ho MPH${ }^{4}$, Monica Chan MRCP ${ }^{2,3}$, Tsin Wen Yeo PhD ${ }^{2,3}$, \\ Charmaine Malenab Manauis MD ${ }^{5}$, Hou Ang MRCS ${ }^{5}$, Partha Pratim De FRCPath ${ }^{6}$, Brenda Sze Peng Ang MPH ${ }^{3,7}$ \\ and Angela Li Ping Chow PhD ${ }^{3,4}$ \\ ${ }^{1}$ Department of Clinical Epidemiology, Tan Tock Seng Hospital, Singapore, ${ }^{2}$ Infectious Disease Department, Tan Tock Seng Hospital, Singapore, ${ }^{3} \mathrm{National}$ Centre for \\ Infectious Diseases, Singapore, ${ }^{4}$ Department of Clinical Epidemiology, Tan Tock Seng Hospital, Singapore, ${ }^{5}$ Emergency Department, Tan Tock Seng Hospital, \\ Singapore, ${ }^{6}$ Department of Laboratory Medicine, Tan Tock Seng Hospital, Singapore and ${ }^{7}$ Infection Prevention and Control Unit, Tan Tock Seng Hospital, Singapore
}

To the Editor-Since monkeypox was first identified in humans in the Democratic Republic of Congo in 1970, most human monkeypox cases have been reported in Central and West Africa, with the largest documented outbreak occurring in

Author for correspondence: Win Mar Kyaw, Email: mar_kyaw_win@ttsh.com.sg Cite this article: Kyaw WM, et al. (2020). Monitoring healthcare professionals after monkeypox exposure: Experience from the first case imported to Asia. Infection Control \& Hospital Epidemiology, 41: 373-375, https://doi.org/10.1017/ice.2019.362
Nigeria in 2017. ${ }^{1}$ Monkeypox, a rare viral zoonotic re-emerging disease caused by an orthopoxvirus, has similar clinical signs and symptoms as smallpox and a case-fatality rate of $11 \%$ in unvaccinated patients. ${ }^{2}$ It can be transmitted from person to person via direct contact with infected lesions, through respiratory secretions, or from contaminated objects and environments. Risk of infection for healthcare workers (HCWs) are high, ${ }^{3}$ and patient-to-HCW transmission of monkeypox has been reported in the Central African Republic and the United Kingdom, where staff used 
Table 1. Categories of Healthcare Workers (HCWs) by Location, Type of Contact, and Type of Personal Protective Equipment

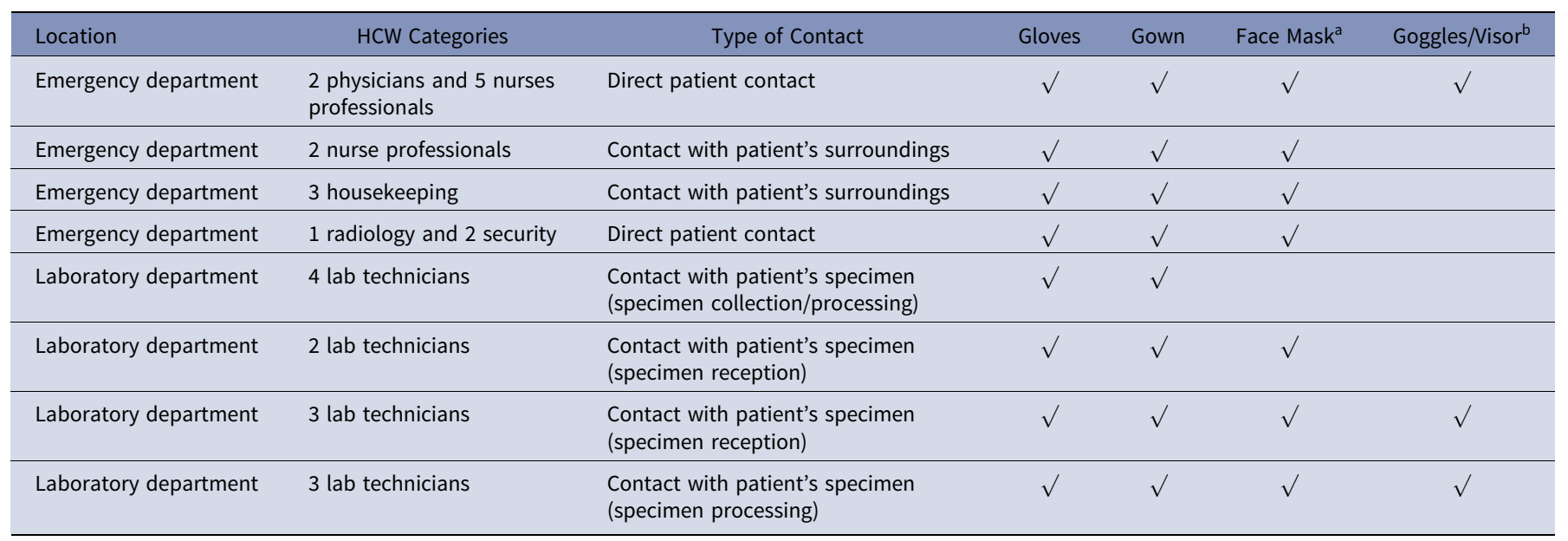

${ }^{\text {aN95 }}$ respirator for respiratory protection.

beye protection.

inadequate standard contact precautions. ${ }^{4-6}$ The Centers for Disease Control and Prevention has recommended standard and contact precautions for the management of human monkeypox. ${ }^{7}$

Singapore, an island city-state in Southeast Asia, is a major travel hub that received $>5,000$ visitors from Africa between January and May 2019. ${ }^{8}$ On May 8, 2019, the human monkeypox case was confirmed in Singapore in a 38 -year-old Nigerian man who arrived in Singapore on April 28, 2019, to attend a workshop. Before his travel to Singapore, he had resided and worked in the Delta state in Nigeria and had attended a wedding on April 21 in a village in Ebonyi State, Nigeria, where he consumed bushmeat. ${ }^{9}$ He presented to the emergency department of Tan Tock Seng Hospital on May 7 with fever, muscle aches, and vesicular skin lesions. Due to his travel history, he was transferred from the ambulance directly to a negative air pressure (NEP) isolation room at the emergency department. After staying for 5 hours at the emergency department, he was admitted into an NEP in an isolation unit at the adjoining National Centre for Infectious Diseases for further clinical management on the same day, and laboratory confirmed as monkeypox infection the next day (May 8).

Contact tracing operations were carried out at the hospital to identify HCWs who were in contact with the patient before admission to the isolation unit. All staff identified as close contacts were assessed for types of personal protective equipment (PPE) used. Laboratory staff who processed the patient's specimens on an open bench (ie, outside a biosafety cabinet) were also considered as having close contact and assessed. Each HCW identified as having possible exposure was contacted via phone call and interviewed by a designated staff to verify the type of contact with the patient and the PPE used.

Following individual risk assessments, $27 \mathrm{HCW}$ s were identified to have had close contact. Of these, $12 \mathrm{HCW}$ s had had direct contact with the patient himself or the patient's surrounding (defined as within $2 \mathrm{~m}$ of the patient) at the emergency department, 3 HCWs had handled the patient's linen and cleaned the NEP room in the emergency department, and 12 were laboratory staff who had handled the patient's specimens. All had protected exposure to the patient, with the appropriate and adequate use of PPE (Table 1).

A designated group of public-health-trained staff implemented follow-up phone surveillance on all staff contacts. Phone calls were made to all nonphysician HCW contacts every other day from the day 1 to day 21 postexposure to monitor their health status. After the initial phone call, the 2 physicians were advised to monitor their own health. Symptoms monitored included fever, swollen lymph nodes, skin rash, headache, and myalgia. All exposed HCWs were also given the phone numbers of the surveillance team for immediate contact if they felt acutely ill. Unwell HCWs were immediately referred to the dedicated infectious disease clinic with appropriate precautions in place for review at the earliest available time. Because the risk of exposure was ascertained to be low for all staff contacts, they were allowed to continue with their routine activities during the surveillance period.

During the follow-up period of 21 days, 2 nursing staff reported respiratory symptoms. They were reviewed by infectious disease physicians and were clinically diagnosed with viral upper respiratory infections. They were treated symptomatically, were given medical leave to rest, and recovered uneventfully. At the end of the surveillance period, none of the 27 HCWs developed symptoms suggestive of monkeypox infection.

We have comprehensively and systematically documented the contact tracing processes and active surveillance activities in a tertiary-care hospital in response to a human monkeypox case importation. A well-developed protocol that enables the early detection of suspected cases of emerging infectious diseases ensured that patients are managed in appropriate isolation room facilities in the emergency department from the outset; this would greatly minimize exposure in a crowded emergency department. Furthermore, clear infection prevention guidelines on the appropriate PPE for different HCWs, based on patient care activities and the transmission risk, are crucial. All HCWs who had attended to the patient had complied with the hospital's infection prevention guidelines. Finally, although the risk of transmission of monkeypox to the HCWs was deemed to be extremely low, we took additional measures to actively follow up on each HCW contact to provide assurance and health education to anxious staff who did not have a good understanding of monkeypox. Early detection of symptoms in close contacts through active phone surveillance may facilitate prompt medical review and diagnosis of new infections to prevent further transmission.

Acknowledgments. Authors acknowledge all staff involved in contact tracing operations at the hospital. 
Financial support. No financial support was provided relevant to this manuscript.

Conflicts of interest. All authors report no conflicts of interest relevant to this manuscript.

\section{References}

1. Ogoina D, Izibewule JH, Ogunleye A, et al. The 2017 human monkeypox outbreak in Nigeria-report of outbreak experience and response in the Niger Delta University Teaching Hospital, Bayelsa State, Nigeria. PloS One 2019;14: e0214229.

2. Ježek Z, Szczeniowski M, Paluku K, Mutombo M. Human monkeypox: clinical features of 282 patients. J Infect Dis 1987;156:293-298.

3. Petersen BW, Kabamba J, McCollum AM, et al. Vaccinating against monkeypox in the Democratic Republic of the Congo. Antivir Res 2019;162:171-177.

4. Nakoune E, Lampaert E, Ndjapou SG, et al. A nosocomial outbreak of human monkeypox in the Central African Republic. Open Forum Infect Dis 2017;4(4):ofx168
5. Petersen E, Abubakar I, Ihekweazu C, et al. Monkeypox-enhancing public health preparedness for an emerging lethal human zoonotic epidemic threat in the wake of the smallpox post-eradication era. Int J Infect Dis 2019;78:78-84.

6. Senthilingam M. Third case of monkeypox reported in the UK, in health care worker CNN website. https://edition.cnn.com/2018/09/26/health/ third-monkeypox-case-uk-intl/index.html. Accessed November 16, 2019.

7. Guideline for isolation precautions: preventing transmission of infectious agents in healthcare settings. Centers for Disease Control and Prevention website. https://www.cdc.gov/infectioncontrol/guidelines/isolation/index. html. Published 2007. Accessed November 16, 2019.

8. Monthly international visitor arrivals. Singapore Tourism Board website. https://www.stb.gov.sg/content/stb/en/statistics-and-market-insights/tourismstatistics/international-visitorarrivals.html. Accessed November 16, 2019.

9. Monkeypox-Singapore. World Health Organization website. https://www. who.int/csr/don/16-may-2019-monkeypox-singapore/en/. Accessed November 16, 2019.

\title{
Methods of a study of terminal cleaning of patient rooms
}

\author{
John D. Coppin BS, MPH${ }^{1}$, Frank C. Villamaria BS, MPH, MS ${ }^{1}$, Marjory D. Williams PhD, RN ${ }^{1,2}$, Laurel A. Copeland PhD ${ }^{3}$, \\ John E. Zeber $\mathrm{PhD}^{1,4}$ and Chetan Jinadatha MD, MPH ${ }^{5,6}$ (D) \\ ${ }^{1}$ Department of Research, Central Texas Veterans' Healthcare System, Temple, Texas, '2Department of Nursing, Central Texas Veterans' Health Care System, \\ Temple, Texas, ${ }^{3}$ Veterans' Affairs Central Western Massachusetts Healthcare System, Leeds, Massachusetts, ${ }^{4}$ University of Massachusetts Amherst School of \\ Public Health \& Health Science, Amherst, Massachusetts, ${ }^{5}$ Department of Medicine, Central Texas Veterans' Health Care System, Temple, Texas and ${ }^{6}$ College \\ of Medicine, Texas A\&M Health Science Center, Bryan, Texas
}

To the Editor-It is encouraging to see that people have reviewed our article "Increased Time Spent on Terminal Cleaning of Patient Rooms May Not Improve Disinfection of High-Touch Surfaces." ${ }^{1}$ However, a related Letter to the Editor raises concerns that some may be misinterpreting both the thrust of our paper and our study methodology. ${ }^{2}$

In our pragmatic report, we aimed to promote better cleaning by presenting research results that suggest that more than adequate time spent on terminal cleaning may not result in additionally lower bioburden on high-touch surfaces. We hope this information will cause practitioners to focus on other important factors such as proper training for environmental services staff (EVS), proper use of appropriate chemicals, and targeting high-touch surfaces that pose the greatest risk for transmission of pathogens to patients. We reiterated that adequate cleaning time is crucial, and we certainly do not advocate taking any shortcuts in the terminal cleaning process. Yet, as in many things, it is the quality of the process not the quantity that counts.

As to methodology, EVS were well-trained and experienced, and they voluntarily collaborated on the project. They were instructed to follow the manufacturer's guidelines for application and contact time. We did not monitor EVS during room cleaning to avoid the Hawthorne effect and to obtain data on unmonitored cleaning.

The 5 high-touch surfaces chosen were the highest-touch surfaces according to published papers at the time of the study. ${ }^{3} \mathrm{We}$ omitted details on the culture process and instead referenced a prior paper. ${ }^{4}$

Author for correspondence: Chetan Jinadatha, E-mail: chetan.jinadatha@va.gov

Cite this article: Coppin JD, et al. (2020). Methods of a study of terminal cleaning of patient rooms. Infection Control \& Hospital Epidemiology, 41: 375-376, https://doi.org/ $10.1017 /$ ice.2019.361
Our analysis plan followed best practices for analyzing count data: use a generalized linear model with appropriate choice of family and link function, and avoid log transforming the data. ${ }^{5}$ We used Bayesian models and reported uncertainty in our estimates, rather than rely on a p-value. Recent articles highlight the pitfalls of statistical significance, which can be particularly problematic in small observational studies without preregistration. ${ }^{6}$ Major journals are now requiring some form of uncertainty interval rather than $P$ values. ${ }^{7}$ We also chose to include model estimates on the actual outcome scale. This makes interpretation easy for those familiar with the outcome (ABC counts from press plates) but not familiar with statistical terminology like incident rate ratios. Our goal was to apply the best methods of analysis and interpretation.

Finally, we provided a full financial support disclosure statement in our article. The salary support for this study was provided by the authors' employers.

Acknowledgments. The views expressed in this article are those of the authors and do not necessarily represent the views of the Department of Veterans' Affairs. Xenex Healthcare Service did not participate in study design or in the collection, analysis, and interpretation of data or in the writing of the report or in the decision to submit the paper for publication.

Financial support. This study was supported by a merit review grant from the Department of Veterans' Affairs to J.Z. (grant no. IIR 12-347), and laboratory activity was supported by a grant from Xenex Healthcare Services, with additional support from the Central Texas Veterans' Health Care System (Temple, TX), Scott \& White Healthcare (Temple, TX), and the jointly sponsored Center for Applied Health Research (Temple, TX).

Conflicts of interest. All authors declare no competing interests. 